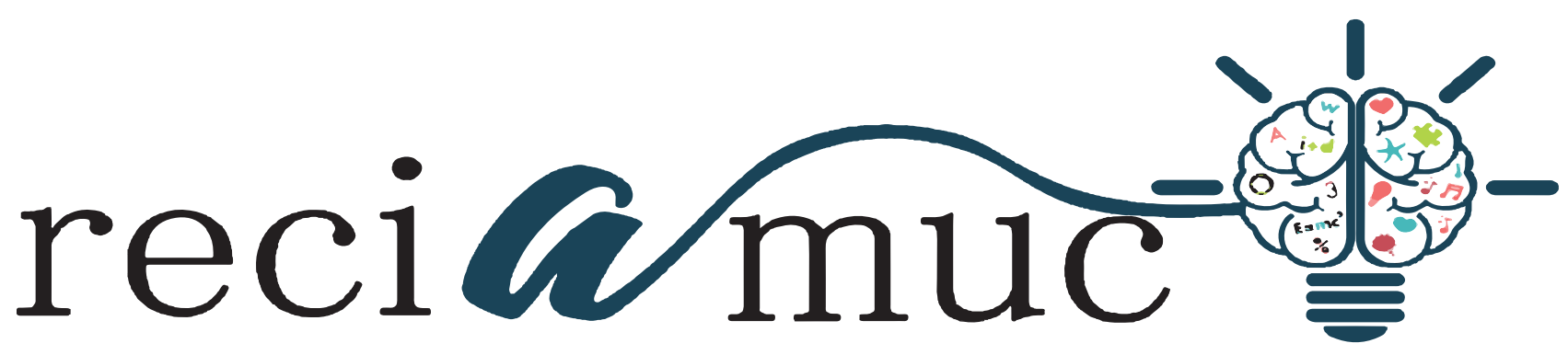

DOI: 10.26820/reciamuc/4.(3).julio.2020.38-49

URL: https://reciamuc.com/index.php/RECIAMUC/article/view/497

EDITORIAL: Saberes del Conocimiento

REVISTA: RECIAMUC

ISSN: 2588-0748

TIPO DE INVESTIGACIÓN: Artículo de Revisión

Código UNESCO: 32 Ciencias Médicas; 3201 Ciencias Clínicas

PAGINAS: $38-49$

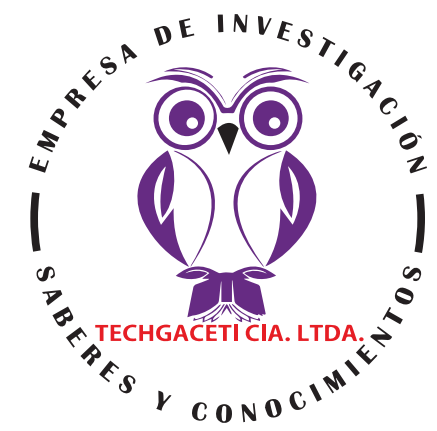

\title{
La psoriasis como desorden común de la piel. Causas y tratamientos
}

Psoriasis as a common skin disorder. Causes and treatments

Psoríase como um distúrbio cutâneo comum. Causas e tratamentos

\begin{abstract}
Katherine Alexandra Carranza Delgado'; Antonella Fanny Montenegro Villavicencio2; Joselyn Lissette Navarrete Moncayo3; Blanca Rebeca Sarmiento Hidalgo ${ }^{4}$
\end{abstract}

RECIBIDO: 18/01/2020 ACEPTADO: 20/03/2020 PUBLICADO: 30/04/2020

1. Médico General; Máster en Prevención de Riesgos Laborales; Investigador Independiente; Guayaquil, Ecuador; dra.katherine.carranza@hotmail.com; (iD) https://orcid.org/0000-0003-0182-1727

2. Médico General; Investigador Independiente; Guayaquil, Ecuador; anto.montenegrov@gmail.com; (iD https://orcid. org/0000-0002-5758-3994

3. Médico General; Investigador Independiente; Guayaquil, Ecuador; joselynnavarrete_94@hotmail.com; (DD https://orcid.org/0000-0002-4608-898X

4. Médico General; Investigador Independiente; Guayaquil, Ecuador; blasar.2987@gmail.com; (iD https://orcid.org/00000002-6239-9710

\author{
CORRESPONDENCIA \\ Katherine Alexandra Carranza Delgado \\ dra.katherine.carranza@hotmail.com
}

Guayaquil, Ecuador 


\section{RESUMEN}

La psoriasis es una enfermedad crónica, mediada por el sistema inmune, caracterizada por el desarrollo de placas cutáneas eritematosas, endurecidas, escamosas, pruríticas y a menudo dolorosas. La patogenia de la psoriasis es impulsada por las citocinas proinflamatorias y la psoriasis se asocia con un mayor riesgo de comorbilidades, que incluyen, entre otras, artritis psoriásica, enfermedad cardiovascular, diabetes mellitus, obesidad, enfermedad inflamatoria intestinal y enfermedad del hígado graso no alcohólico en comparación con la población general. Este articulo explora la relación fisiopatológica entre la psoriasis y sus comorbilidades comunes y discute la necesidad de nuevos paradigmas de tratamiento que incluyan estrategias para reducir la inflamación sistémica en pacientes con psoriasis moderada a severa.La evidencia actual sugiere que prevenir el daño asociado con la inflamación y prevenir el desarrollo de futuros daños inflamatorios y comorbilidades, puede ser un objetivo de tratamiento potencialmente alcanzable para muchos pacientes con psoriasis en placas de moderada a severa cuando las terapias biológicas se utilizan temprano en la enfermedad. Los resultados de los estudios prospectivos en curso sobre los efectos de la biología en los marcadores de inflamación sistémica en pacientes con psoriasis fortalecerán la base de evidencia clínica que puede usarse para informar las decisiones de tratamiento para pacientes con psoriasis moderada a severa. La psoriasis es una enfermedad inflamatoria sistémica y se necesitan tratamientos para optimizar los resultados del paciente.

Palabras clave: Psoriasis, Inflamación, Enfermedad crónica de la piel.

\section{ABSTRACT}

Psoriasis is a chronic disease, mediated by the immune system, characterized by the development of erythematous, hardened, scaly, pruritic, and often painful skin plaques. The pathogenesis of psoriasis is driven by proinflammatory cytokines, and psoriasis is associated with an increased risk of comorbidities, including but not limited to psoriatic arthritis, cardiovascular disease, diabetes mellitus, obesity, inflammatory bowel disease, and nonalcoholic fatty liver disease. compared to the general population. This article explores the pathophysiological relationship between psoriasis and its common comorbidities and discusses the need for new treatment paradigms that include strategies to reduce systemic inflammation in patients with moderate to severe psoriasis. Current evidence suggests that preventing the damage associated with inflammation and preventing the development of future inflammatory damage and comorbidities, may be a potentially achievable treatment target for many patients with moderate to severe plaque psoriasis when biological therapies are used early in the illness. The results of ongoing prospective studies on the effects of biology on markers of systemic inflammation in patients with psoriasis will strengthen the clinical evidence base that can be used to inform treatment decisions for patients with moderate to severe psoriasis. Psoriasis is a systemic inflammatory disease and treatments are needed to optimize patient outcomes.

Keywords: Psoriasis, Inflammation, Chronic skin disease.

\section{RESUMO}

A psoríase é uma doença crônica, mediada pelo sistema imunológico, caracterizada pelo desenvolvimento de placas cutâneas eritematosas, endurecidas, escamosas, pruriginosas e frequentemente dolorosas. A patogênese da psoríase é causada por citocinas pró-inflamatórias, e a psoríase está associada a um risco aumentado de comorbidades, incluindo, mas não se limitando a, artrite psoriática, doença cardiovascular, diabetes mellitus, obesidade, doença inflamatória intestinal e doença hepática gordurosa não alcoólica. em comparação com a população em geral. Este artigo explora a relação fisiopatológica entre a psoríase e suas comorbidades comuns e discute a necessidade de novos paradigmas de tratamento que incluam estratégias para reduzir a inflamação sistêmica em pacientes com psoríase moderada a grave. As evidências atuais sugerem que prevenir os danos associados à inflamação e impedir o desenvolvimento de futuros danos e comorbidades inflamatórias, pode ser um alvo de tratamento potencialmente alcançável para muitos pacientes com psoríase em placas moderada a grave quando terapias biológicas são usadas no início da doença. Os resultados de estudos prospectivos em andamento sobre os efeitos da biologia nos marcadores de inflamação sistêmica em pacientes com psoríase fortalecerão a base de evidências clínicas que podem ser usadas para informar as decisões de tratamento para pacientes com psoríase moderada a grave. A psoríase é uma doença inflamatória sistêmica e são necessários tratamentos para otimizar os resultados dos pacientes.

Palavras-chave: Psoríase, inflamação, doença de pele crônica. 


\section{Introducción}

La psoriasis es una afección inflamatoria crónica mediada por el sistema inmunitario que afecta aproximadamente al $3 \%$ de los adultos y al $0,1 \%$ de los niños y adolescentes en los países desarrollados(Paller, Singh, \& Cloutier, 2018). Se caracteriza por placas eritematosas bien delimitadas cubiertas por escamas de color blanco plateado, que generalmente se presentan en una distribución simétrica que involucra los codos, las rodillas, el tronco y el cuero cabelludo. El inicio de la psoriasis se desencadena cuando los factores genéticos y / o ambientales activan las células dendríticas plasmacitoides, lo que resulta en la producción de numerosas citocinas proinflamatorias, incluido el factor de necrosis tumoral (TNF) -a, interferón (IFN) -c, interleucina (IL) -17, IL-22, IL-23 e IL-1b. Muchas de estas citocinas estimulan la hiperproliferación de queratinocitos, que perpetúa un ciclo de inflamación crónica(Swindell, Johnston, \& Xing, 2013).

En la psoriasis de moderada a grave, los niveles elevados de múltiples citocinas proinflamatorias se encuentran no solo en lesiones cutáneas, sino también en la sangre. Las elevaciones sistémicas en estas citocinas promueven la inflamación subclínica crónica (inflamación asintomática que puede causar daño tisular con el tiempo ) asociado con comorbilidades que afectan de manera desproporcionada a los pacientes con psoriasis, incluida la artritis psoriásica (PsA), la enfermedad cardiovascular (ECV), la diabetes mellitus, la obesidad, la enfermedad inflamatoria intestinal y la enfermedad del hígado graso no alcohólico (NAFLD) (tabla 1) (Egeberg, Mallbris, Warren, \& RB, 2016).

Se presume que el tratamiento sistémico temprano dirigido a las citocinas proinflamatorias asociadas con la patogénesis de la psoriasis no solo mejorará los síntomas cutáneos sino que también reducirá la inflamación sistémica, por lo tanto, mejorará los resultados a largo plazo a través de la mitigación de la progresión de la comorbilidad. Esta revisión explora la relación fisiopatológica entre la psoriasis y sus comorbilidades comunes, y analiza la necesidad de nuevos paradigmas de tratamiento que incluyan estrategias para reducir los efectos de la inflamación sistémica en la psoriasis en placas de moderada a severa.

\section{Metodología}

Para el desarrollo de este proceso investigativo, se plantea como metodología la encaminada hacia una orientación científica particular que se encuentra determinada por la necesidad de indagar en forma precisa y coherente una situación, en tal sentido (Davila, 2015) define la metodología "como aquellos pasos previos que son seleccionados por el investigador para lograr resultados favorables que le ayuden a plantear nuevas ideas".(p.66)

Lo citado por el autor, lleva a entender que el desarrollo de la acción investigativa busca simplemente coordinar acciones enmarcadas en una revisión bibliográfica con el fin de complementar ideas previas relacionadas a la psoriasis como enfermedad común de la piela través de una revisión de literatura, para así finalmente elaborar un cuerpo de consideraciones generales que ayuden a ampliar el interés propuesto.

\section{Tipo de Investigación}

Dentro de toda práctica investigativa, se precisan acciones de carácter metodológico mediante las cuales, se logra conocer y proyectar los eventos posibles que la determinan, así como las características que hacen del acto científico un proceso interactivo ajustado a una realidad posible de ser interpretada. En este sentido, se puede decir, que la presente investigación corresponde al tipo documental, definido por Castro (2016), "se ocupa del estudio de problemas planteados a nivel teórico, la información requerida para abordarlos se encuentra básicamente en materiales impresos, audiovi- 
suales y /o electrónicos". (p.41).

En consideración a esta definición, la orientación metodológicapermitió la oportunidad de cumplir con una serie de actividades inherentes a la revisión y lectura de diversos documentos donde se encontraron ideas explicitas relacionadas con los tópicos encargados de identificar a cada característica insertada en el estudio. Por lo tanto, se realizaron continuas interpretaciones con el claro propósito de revisar aquellas apreciaciones o investigaciones propuestas por diferentes investigadores relacionadas con el tema de interés, para luego dar la respectiva argumentación a los planteamientos, en función a las necesidades encontradas en la indagación.

\section{Fuentes Documentales}

El análisis correspondiente a las características que predomina en el tema seleccionado, llevan a incluir diferentes fuentes documentales encargadas de darle el respectivo apoyo y en ese sentido cumplir con la valoración de los hechos a fin de generar nuevos criterios que sirven de referencia a otros procesos investigativos. Para (CASTRO, 2016) las fuentes documentales incorporadas en la investigación documental o bibliográfica, "representa la suma de materiales sistemáticos que son revisados en forma rigurosa y profunda para llegar a un análisis del fenómeno".(p.41). Por lo tanto, se procedió a cumplir con la realización de una lectura previa determinada para encontrar aquellos aspectos estrechamente vinculados con el tema, con el fin de explicar mediante un desarrollo las respectivas apreciaciones generales de importancia.

\section{Técnicas para la Recolección de la Infor- mación}

La conducción de la investigación para ser realizada en función a las particularidades que determinan a los estudios documentales, tiene como fin el desarrollo de un conjunto de acciones encargadas de llevar a la selección de técnicas estrechamente vin- culadas con las características del estudio. En tal sentido, (Bolívar, 2015), refiere, que es "una técnica particular para aportar ayuda a los procedimientos de selección de las ideas primarias y secundarias". (p. 71).

Por ello, se procedió a la utilización del subrayado, resúmenes, fichaje, como parte básica para la revisión y selección de los documentos que presentan el contenido teórico. Es decir, que mediante la aplicación de estas técnicas se pudo llegar a recoger informaciones en cuanto a la revisión bibliográfica de los diversos elementos encargados de orientar el proceso de investigación. Tal como lo expresa, (Bolívar, 2015) "las técnicas documentales proporcionan las herramientas esenciales y determinantes para responder a los objetivos formulados y llegar a resultados efectivos" (p. 58). Es decir, para responder con eficiencia a las necesidades investigativas, se introdujeron como técnica de recolección el método inductivo, que hizo posible llevar a cabo una valoración de los hechos de forma particular para llegar a la explicación desde una visión general.

Asimismo, se emplearon las técnicas de análisis de información para la realización de la investigación que fue ejecutada bajo la dinámica de aplicar diversos elementos encargados de determinar el camino a recorrer por el estudio, según, (Bolívar, 2015) las técnicas de procesamiento de datos en los estudios documentales "son las encargadas de ofrecer al investigador la visión o pasos que debe cumplir durante su ejercicio, cada una de ellas debe estar en correspondencia con el nivel a emplear" (p. 123). Esto indica, que para llevar a cabo el procesamiento de los datos obtenidos una vez aplicado las técnicas seleccionadas, tales como: fichas de resumen, textual, registros descriptivos entre otros, los mismos se deben ajustar al nivel que ha sido seleccionado. 


\section{CARRANZA DELGADO, K., MONTENEGRO VILLAVICENCIO, A., NAVARRETE MONCAYO, J., \& SARMIENTO}

HIDALGO, B.

\section{Resultados}

\section{Psoriasis: una enfermedad inflamatoria} sistémica

Históricamente, la psoriasis se consideraba una enfermedad que se limitaba a la piel y generalmente se trataba con agentes tópicos o fototerapia. Aunque tales terapias pueden proporcionar un alivio efectivo de los síntomas localizados de la piel, hacen poco para afectar las causas subyacentes de la enfermedad. Con los avances recientes en la comprensión de la naturaleza inflamatoria de la psoriasis, los esfuerzos de investigación se han centrado en dilucidar los roles de las citocinas proinflamatorias específicas que contribuyen a enfermedad patogénica, con el objetivo de desarrollar nuevas terapias dirigidas(Takeshita, Grewal, \& Langan, 2017).

La psoriasis se desarrolla cuando las células dendríticas plasmocitoides activadas producen la citocina proinflamatoria IFN-a, que activa las células dendríticas mieloides junto con IFN-c, TNF-a, IL-1b e IL-6. Estas células dendríticas mieloides activadas producen IL-12 e IL- 23, que activan las células T helper (Th) 1 y Th17 correspondientes (Nestle, Kaplan, \& Barker, 2009). Una vez iniciado, este ciclo de inflamación continúa de manera crónica, ya que las células Th1 activadas producen TNF-a y las células Th17 producen IL-17A, IL-17F e IL-22. Las citocinas activan aún más los queratinocitos que producen una variedad de citocinas, quimiocinas y péptidos antimicrobianos que promueven una respuesta proinflamatoria continua (Nestle, Kaplan, \& Barker, 2009).

A medida que progresa la psoriasis, su naturaleza sistémica se evidencia por el aumento de los niveles séricos de múltiples citocinas proinflamatorias, incluidos TNF-a, IFN-C, IL-6, IL-8, IL-12, IL-17A e IL-18, en pacientes con psoriasis en comparación con controles sanos(Kolbinger, Loesche, \& Valentin, 2017). Las observaciones de los estudios de tomografía por emisión de positrones con 18F-fluorodeoxiglucosa / tomo- grafía computarizada (FDG PET / CT) también validan la hipótesis de que la psoriasis es una enfermedad inflamatoria sistémica. En estos estudios, los pacientes con psoriasis moderada a severa muestran inflamación subclínica en el hígado, articulaciones y tendones, además de un aumento significativo de la inflamación arterial y subcutánea global, y los pacientes con psoriasis leve tenían inflamación subclínica en la aorta (Hjuler, Gormsen, \& Vendelbo, 2017).

También, el ultrasonido de las arterias femorales puede mejorar la detección de la aterosclerosis subclínica en pacientes con psoriasis moderada a severa y la resistencia a la insulina ayuda a proporcionar una mejor predicción de la aterosclerosis subclínica que los factores de riesgo de ECV tradicionales (Gonzalez-Cantero, Gonzalez-Cantero, \& Sanchez-Moya, 2019). Estas observaciones han llevado a una mejor comprensión de cómo un subconjunto de moléculas inflamatorias se difunde en la circulación sistémica y luego a varios sistemas de órganos; esto puede contribuir a la patología de las comorbilidades inflamatorias comunes en la psoriasis (Fig. 1) (Lowes, Suarez-Farinas, \& Krueger, 2014) La Tabla 1 destaca los hallazgos de estudios recientes que muestran que las vías inflamatorias sistémicas compartidas contribuyen a la patogénesis de la psoriasis y sus comorbilidades. 
Tabla 1. Comorbilidades asociadas con psoriasis

\begin{tabular}{|c|c|}
\hline Comorbilidad & Mayor riesgo con psoriasis \\
\hline $\mathrm{CVD}^{77}$ & 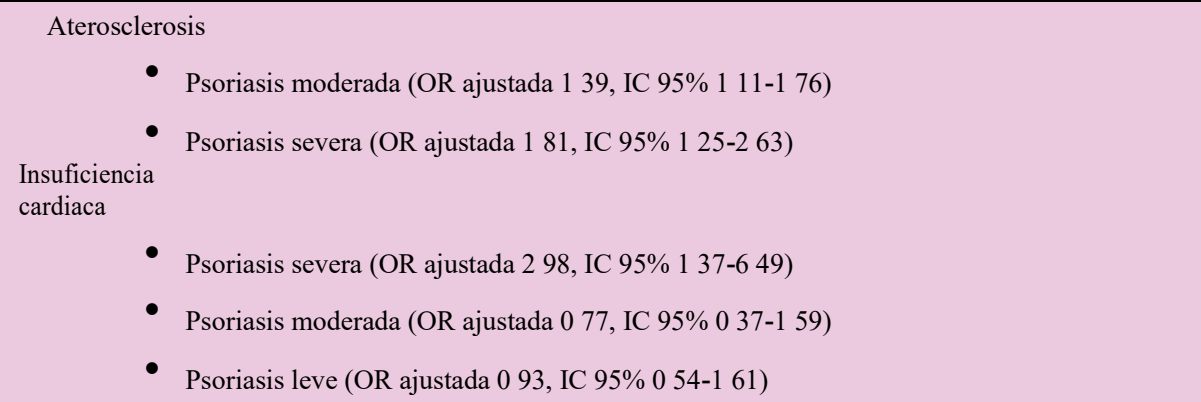 \\
\hline Diabetes mellitus ${ }^{78}$ & Psoriasis leve (TIR 1 49, IC 95\% 1 43-1 56) \\
\hline 79 & Psoriasis severa (TIR 2 13, IC 95\% 1 91-2 37) \\
\hline & $\begin{array}{l}\text { Psoriasis leve (agrupada OR } 1 \text { 46, IC 95\% } 117-1 \text { 82) } \\
\text { Psoriasis de moderada a severa (agrupada OR } 2 \text { 23, IC 95\% } 1 \text { 63-3 05) }\end{array}$ \\
\hline NAFLD & En general (OR 2 15, IC 95\% 1 57-2 94) \\
\hline $\begin{array}{r}{ }^{80} \\
\text { PsA } \\
\text { EII }^{81}\end{array}$ & $\begin{array}{l}\text { Psoriasis de moderada a severa (OR } 207 \text {, IC } 95 \% 159-271 \text { ) } \\
\text { Afecta el } \\
0 \% \text { de pacientes con psoriasis } \\
\text { Los pacientes con psoriasis tienen un riesgo } 29 \text { veces mayor de desarrollar enfermedad de Crohn que la } \\
\text { población general }\end{array}$ \\
\hline
\end{tabular}

Fuente: (Nestle, Kaplan, \& Barker, 2009)

\section{Metas para tratar la inflamación sistémica en la psoriasis}

Los estudios en otras enfermedades inflamatorias inmunomediadas (IMID), incluida la enfermedad de Crohn y la artritis reumatoide (AR), han demostrado los beneficios significativos del tratamiento temprano con productos biológicos aprobados para mejorar los resultados y sugieren que enfoques similares pueden ser útiles para controlar la inflamación sistémica y optimizar Resultados a largo plazo en la psoriasis(Girolomoni, Griffiths, \& Krueger, 2015). En particular, varios de los mismos agentes biológicos están aprobados para el tratamiento de la psoriasis moderada a severa y la AR (etanercept, adalimumab, certolizumab e infliximab) y / o enfermedad de Crohn (adalimumab, infliximab, certolizumab y ustekinumab) debido a la centralidad de sus objetivos en la patogénesis de la enfermedad.

A medida que los profesionales reconocen más fácilmente la psoriasis como una enfermedad sistémica y ponen más énfasis en controlar la inflamación sistémica, los objetivos del tratamiento se pueden separar en dos categorías distintas según la viabilidad de lograr los resultados deseados. El primer objetivo, prácticamente implementable, es potencialmente prevenir el daño asociado con la inflamación sistémica y, al mismo tiempo, prevenir potencialmente la progresión de la psoriasis y sus comorbilidades. El segundo objetivo, quizás más elevado y más progresista, es potencialmente revertir el daño inflamatorio existente y los signos y síntomas de comorbilidades.

Objetivo 1: prevenir el daño asociado con la inflamación y prevenir futuros daños / comorbilidades

Se han identificado numerosos biomarcadores de inflamación. Algunos de los marcadores de daño inflamatorio y riesgo cardiovascular más comúnmente utilizados en la psoriasis activa incluyen la proteína C reactiva (PCR) y la tasa de sedimentación globular (VSG) (Gabay \& Kushner, 1999). Los niveles de PCR están positivamente co- 
rrelacionados con la gravedad de la enfermedad. medido por el área de psoriasis y el índice de gravedad (PASI).

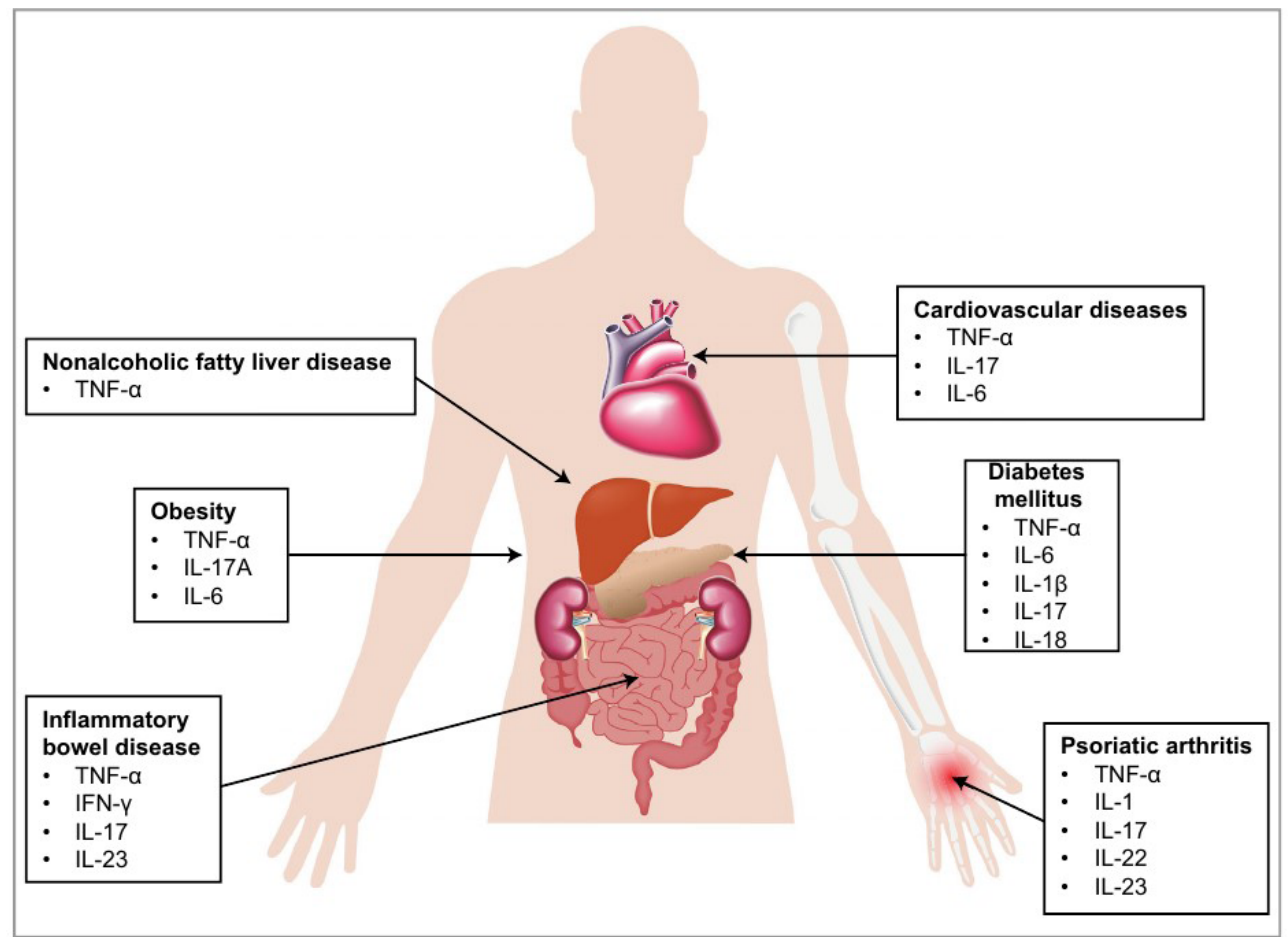

Gráfico 1. Psoriasis. Comorbilidades y citocinas inflamatorias clave. IFN, interferón; IL, interleucina; TNF, factor de necrosis tumoral.

Fuente: (Swindell, Johnston, \& Xing, 2013)

La PCR también es un predictor independiente del riesgo de ECV y está implicada en el desarrollo de lesiones ateroscleróticas porque reduce la expresión de óxido nítrico sintasa y prostaciclina sintasa, une el colesterol de lipoproteína de baja densidad que estimula su absorción por los macrófagos y aumenta la regulación expresión de moléculas de adhesión en células endoteliales(Shrivastava, Singh, Raizada, \& Singh, 2015). A medida que disminuyen los niveles de PCR, el riesgo cardiovascular disminuye. Sin embargo, existe evidencia que cuestiona la utilidad clínica de la PCR para evaluar el riesgo cardiovascular en individuos con afecciones inflamatorias como la psoriasis, lo que indica una necesidad de alternativas. Biomarcadores de riesgo de ECV en pacientes con afecciones inflamatorias subyacentes (Joshi, Lerman, \& Aberra, 2016).
El tratamiento con agentes biológicos disminuye la inflamación sistémica medida por los niveles de ESR y CRP en varios estados de enfermedad diferentes. Por ejemplo, los inhibidores de TNF-a reducen significativamente los niveles de ESR y CRP en la AR. Los inhibidores de TNF-a también reducen significativamente los niveles de CRP en pacientes con síndrome metabólico o enfermedad de Crohn(Hanauer, Sandborn, \& Rutgeerts, 2006). De manera similar, la IL12 / 23 el inhibidor ustekinumab reduce los niveles de ESR y CRP en la enfermedad de Crohn, y el inhibidor de IL-17A secukinumab reduce los niveles de CRP en la espondilitis anquilosante y reduce los niveles de ESR en PsA. En pacientes con psoriasis moderada a severa tratados con terapias sistémicas, incluyendo metotrexato, adalimumab, etanercept, infliximab e ixekizumab, los estudios han reportado reducciones en los ni- 
veles de ESR y / o PCR (Gisondi, Lora, \& Bonauguri, 2013).

Los datos de estudios retrospectivos respaldan el concepto de que ciertos agentes biológicos dirigidos a las citocinas proinflamatorias relevantes involucradas en la patogénesis de la psoriasis pueden ser las mejores opciones de tratamiento para reducir la probabilidad de que los pacientes con psoriasis desarrollen ECV. Un gran análisis retrospectivo de EE. UU. De las tasas de eventos cardiovasculares en pacientes con psoriasis (no se informó la gravedad) encontró que los pacientes que recibían inhibidores de TNF-a (etanercept, infliximab o adalimumab; porcentajes no especificados) tenían riesgos significativamente menores de infarto de miocardio (IM) en comparación con los pacientes recibir terapias tópicas [odds ratio ajustado 0 50, intervalo de confianza (IC) del 95\% 0 32-0 79], y que el tratamiento con estas terapias disminuyó el riesgo de eventos cardiovasculares mayores en comparación con el metotrexato durante 12 meses de seguimiento [ajustado cociente de riesgos (HR) 0 55, $\mathrm{P}<0$ 001] (Wu, Guerin, \& Sundaram, 2017).

Además, durante 24 meses de seguimiento, la exposición acumulativa a los inhibidores de TNF-a se asoció con una reducción del $11 \%$ en el riesgo cardiovascular por cada 6 meses de tratamiento ( $P=0$ 02)(Wu, Guerin, \& Sundaram, 2017). Otra retrospectiva estudio utilizando una base de datos de reclamos administrativos de los Estados Unidos que incluía información de aproximadamente 25 millones de pacientes y sus dependientes, comparó más de 11000 pacientes con psoriasis que recibieron inhibidores de TNF-a con más de 12000 pacientes con psoriasis que fueron tratados con fototerapia(Wu, Guerin, \& Sundaram, 2017). Descubrieron que la cohorte de inhibidores de TNF tuvo un menor riesgo de eventos cardiovasculares mayores en comparación con la cohorte de fototerapia (HR ajustada 0 77, IC 95\% 0 60-0 99; P = 0 046).
Aunque la mayoría de las investigaciones sobre los efectos cardiovasculares del tratamiento con inhibidores del TNF-a en la psoriasis han informado mejoras en los resultados, no todos los estudios sugieren una correlación positiva entre el tratamiento con agentes biológicos y un riesgo cardiovascular reducido. Un estudio retrospectivo de más de 25000 pacientes con psoriasis moderada a severa evaluó a aquellos tratados con terapias sistémicas, incluidos metotrexato, ciclosporina, alefacept, efalizumab, adalimumab, etanercept e infliximab, y los comparó con pacientes que recibieron fototerapia ultravioleta B. En este estudio, no se encontraron diferencias significativas en el riesgo general de infarto de miocardio entre los dos grupos (HR ajustado 133, IC 95\% 0 90-1 96) (Abuabara, Lee, \& Kimball, 2011) 
Tabla 2. Patogenia de las comorbilidades de psoriasis

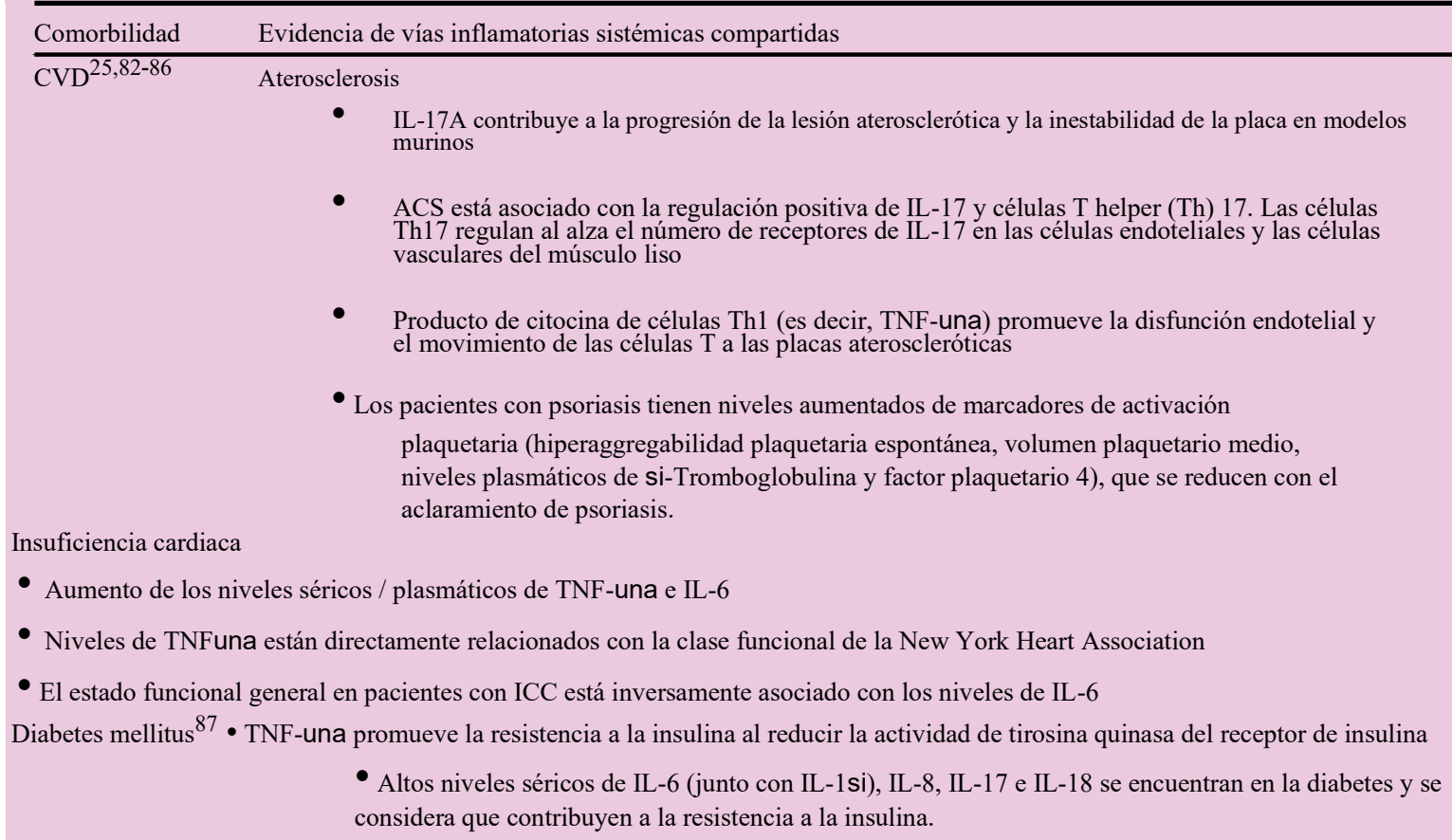

- IL-17A contribuye a la progresión de la lesión aterosclerótica y la inestabilidad de la placa en modelos murinos

- $\quad$ ACS está asociado con la regulación positiva de IL-17 y células T helper (Th) 17. Las células Th17 regulan al alza el número de receptores de IL-17 en las células endoteliales y las células vasculares del músculo liso

- $\quad$ Producto de citocina de células Th1 (es decir, TNF-una) promueve la disfunción endotelial y el movimiento de las células $\mathrm{T}$ a las placas ateroscleróticas

- Los pacientes con psoriasis tienen niveles aumentados de marcadores de activación plaquetaria (hiperaggregabilidad plaquetaria espontánea, volumen plaquetario medio, niveles plasmáticos de si-Tromboglobulina y factor plaquetario 4), que se reducen con el

Insuficiencia cardiaca aclaramiento de psoriasis.

- Aumento de los niveles séricos / plasmáticos de TNF-una e IL-6

- Niveles de TNFuna están directamente relacionados con la clase funcional de la New York Heart Association

- El estado funcional general en pacientes con ICC está inversamente asociado con los niveles de IL-6

Diabetes mellitus ${ }^{87}$ - TNF-una promueve la resistencia a la insulina al reducir la actividad de tirosina quinasa del receptor de insulina

- Altos niveles séricos de IL-6 (junto con IL-1si), IL-8, IL-17 e IL-18 se encuentran en la diabetes y se considera que contribuyen a la resistencia a la insulina.

Obesidad $^{88-90}$

- El tejido adiposo de las personas con obesidad promueve el desarrollo de células Th17

- La expresión de IL-17A está regulada al alza en la obesidad

- Otras citocinas, como TNF-una e IL-6, contribuyen al estado proinflamatorio de la obesidad

NAFLD 9191

- $\quad$ TNF-una exacerba la resistencia a la insulina hepática, lo que resulta en un aumento de la síntesis de FFA y una disminución de la oxidación de FFA, promoviendo así la esteatosis hepática

PsA92-98

- La IL-23 y las citocinas posteriores IL-17 e IL-22 promueven la entesitis en modelos murinos

- $\quad$ Aumento de las concentraciones de células Th17, IL-17RA, IL-17A, TNF-una e IL-1 en la sinovial de pacientes con PsA

- IL-17 e IL-22 vinculadas a la formación de pannus en la articulación, erosión articular y formación de hueso nuevo

EII $^{99} \quad$ enfermedad de Crohn

- Mediado por células Th1 y sus productos de citoquinas (es decir, TNF-una e IFN-C)

- Niveles aumentados de IL-17 e IL-23 encontrados en la lámina intestinal propia de pacientes con enfermedad de Crohn

SCA, síndrome coronario agudo; CHF, insuficiencia cardíaca congestiva; ECV, enfermedad cardiovascular; FFA, ácido graso libre; EII, enfermedad inflamatoria intestinal; IFN, interferón; IL, interleucina; NAFLD, enfermedad del hígado graso no alcohólico; PsA, artritis psoriásica; TNF, factor de necrosis tumoral.

Fuente: (Gisondi, Lora, \& Bonauguri, 2013)

Además, también se han realizado dos pequeños estudios prospectivos de tratamiento con adalimumab en psoriasis moderada a severa. Un estudio evaluó los efectos del adalimumab en comparación con el placebo durante 16 semanas seguido de un tratamiento abierto con adalimumab durante 1 año(Bissonnette, Harel, \& Krueger, 2017). El otro estudio examinó el adalimumab, la fototerapia y el placebo durante 12 sema- nas, seguido de una terapia abierta con adalimumab durante 1 año(Mehta, Shin, \& Joshi, 2018). Ninguno de los dos estudios demostró una inflamación vascular reducida en los pacientes tratados con adalimumab en comparación con placebo, ya sea a las 12 semanas o 16 semanas o con fototerapia a las 12 semanas según lo medido por 18F-FDG PET / CT. Sin embargo, uno de estos estudios evaluó varios biomarcadores 
y encontró niveles disminuidos de acetilación de glucoproteínas, un nuevo biomarcador compuesto de inflamación sistémica, en pacientes tratados con adalimumab en comparación con aquellos tratados con fototerapia.

Además, un pequeño estudio prospectivo de ustekinumab en una población coreana observó una disminución significativa de la inflamación vascular en individuos que lograron una mejora $\geq 75 \%$ en PASI durante un período de tratamiento promedio de 5 meses(Kim, Lee, \& Pak, 2019). Los estudios prospectivos en curso incluyen ensayos de inflamación vascular en psoriasis (VIP) que actualmente evalúan los efectos de ustekinumab (VIP-U; NCT02187172), secukinumab (VIP-S; NCT02690701) y apremilast (VIP-A; NCT03082729) en la inflamación aórtica medida por 18F-FDG PET / CT y en los niveles de biomarcadores asociado con el riesgo metabólico y cardiovascular. Los resultados de los estudios de agentes biológicos en los IMID distintos de la psoriasis respaldan la hipótesis de que los agentes biológicos pueden reducir la inflamación sistémica y prevenir el daño cardiovascular (Low, Symmons, \& Lunt, 2017).

Además de tener el potencial de prevenir el daño asociado con la inflamación vascular y prevenir la ECV, el tratamiento con inhibidores de TNF-a puede jugar un papel importante en la reducción del daño inflamatorio en otras comorbilidades de psoriasis. En pacientes con psoriasis, el aumento de la inflamación sistémica y la desregulación de las adipocitocinas, incluidas la leptina, la resistina y la adiponectina, aumentan el riesgo de resistencia a la insulina que puede progresar al desarrollo de diabetes mellitus y síndrome metabólico(Reich, 2012). Este riesgo aumenta con la severidad de la psoriasis y disminuye con terapia con inhibidores de TNF-a.

La obesidad es una comorbilidad bien conocida de la psoriasis, y en la obesidad, como en la psoriasis y la PsA, existe una desregulación de los niveles y / o funciones de IL, TNF-a y otras adipocytokines. Sin embargo, un posible papel para la biología hasta la fecha no se han observado agentes para mitigar la obesidad en la psoriasis. De hecho, los inhibidores de TNF-a han inducido un aumento de peso moderado en pacientes con psoriasis moderada a severa(Gisondi, Conti, \& Galdo, Ustekinumab does not increase body mass index in patients with chronic plaque psoriasis: a prospective cohort study, 2013). No se ha informado evidencia de aumento de peso clínicamente significativo en estudios de ustekinumab o ixekizumab en psoriasis de moderada a severa.

Objetivo 2: revertir el daño existente / condiciones comórbidas causadas por la inflamación

La evidencia que respalda la reversión del daño existente y / o las condiciones comórbidas causadas por la inflamación sistémica en pacientes con psoriasis es alentadora pero no tan bien desarrollada como la evidencia que respalda el primer objetivo declarado de prevenir daños y prevenir futuras comorbilidades. Un estudio sobre el tratamiento con ustekinumab en pacientes con psoriasis moderada a severa $(\mathrm{N}=46)$ mostró una regresión de las anormalidades sinoviales e inflamatorias subclínicas, Io que sugiere la posibilidad de que el desarrollo biológico de la psoriasis pueda inhibirse mediante el tratamiento biológico de la psoriasis(Savage, Goodfield, Horton, \& L, 2018). Además, un estudio de 105 pacientes con diversos grados de severidad de la psoriasis utilizó la angiografía por TC para mostrar que el tratamiento con agentes sistémicos o biológicos se asoció con una mejora en la carga de placa coronaria no calcificada(Lerman \& Joshi, 2017).

Además, aunque en la mayoría de estos estudios se planteó la hipótesis de que el uso de un tratamiento antiinflamatorio sistémico en la psoriasis conduciría a una disminución de la gravedad de las comorbilidades, 


\section{CARRANZA DELGADO, K., MONTENEGRO VILLAVICENCIO, A., NAVARRETE MONCAYO, J., \& SARMIENTO HIDALGO, B.}

(3)

los estudios publicados hasta la fecha han sido de tamaño y alcance limitados, y los estudios más amplios y bien diseñados necesitaba proporcionar un vínculo definitivo entre este tipo de mejoras y reducciones en la inflamación sistémica. En general, estos hallazgos son alentadores y sugieren que el tratamiento temprano con productos biológicos tiene el potencial, al menos a corto plazo, de revertir el daño causado por las comorbilidades inflamatorias asociadas con la psoriasis.

\section{Conclusión}

Dada la amplitud de los datos que indican que la psoriasis es una enfermedad sistémica y debe tratarse como tal, parece claro que se necesitan tratamientos sistémicos para optimizar los resultados del paciente. Se establecieron dos objetivos para guiar a los profesionales hacia el manejo efectivo de la inflamación sistémica en la psoriasis. Según la evidencia disponible, el primer objetivo, para prevenir el daño asociado con la inflamación y prevenir futuros daños / comorbilidades, parece ser alcanzable para muchos pacientes con el uso de la terapia con agentes biológicos temprano en el curso de la enfermedad, cuyo objetivo es Las citocinas proinflamatorias apropiadas.

El segundo objetivo, más elevado, revertir el daño existente / las condiciones comórbidas causadas por la inflamación, tiene menos evidencia que respalde su alcance. Sin embargo, Los resultados de varios estudios en animales y humanos sugieren que revertir el daño puede ser más factible de lo que los practicantes aprecian actualmente. El avance de la investigación sobre nuevos biomarcadores, que podría mejorar el diagnóstico temprano de comorbilidades - la evaluación clínica de comorbilidades, puede ayudar a los profesionales a evaluar mejor la respuesta del paciente a las terapias sistémicas.

Con el fin de proporcionar un mayor apoyo para la consecución de estos objetivos de tratamiento, se están realizando esfuerzos para recopilar datos prospectivos. Se espera que los resultados de estos ensayos prospectivos brinden información importante sobre el papel que los agentes biológicos pueden desempeñar en el tratamiento de la inflamación sistémica asociada con la psoriasis, lo que podría mejorar el diagnóstico temprano de comorbilidades o la evaluación clínica de comorbilidades, puede ayudar a los profesionales a evaluar mejor la respuesta del paciente a las terapias sistémicas.

\section{Bibliografía}

Abuabara, K., Lee, H., \& Kimball, A. (2011). The effect of systemic psoriasis therapies on the incidence of myocardial infarction: a cohort study. $\mathrm{Br} \mathrm{J}$ Dermatol, 1066-73.

Bissonnette, R., Harel, F., \& Krueger, J. (2017). TNF-a antagonist and vas-cular inflammation in patients with psoriasis vulgaris: a random-ized placebo-controlled study. J Invest Dermatol, 1638-45.

Bolívar, J. (2015). Investigación Documental. México. Pax.

Castro, J. (2016). Técnicas Documentales. México. Limusa.

Davila, A. (2015). Diccionario de Términos Científicos. . Caracas: Editorial Oasis.

Egeberg, A., Mallbris, L., Warren, \& RB. (2016). Association between psori-asis and inflammatory bowel disease: a Danish nationwide cohort study. $\mathrm{Br}$ J Dermatol , 487-92.

Gabay, C., \& Kushner, I. (1999). Acute-phase proteins and other systemic responses to inflammation. N Engl J Med, 448-54.

Girolomoni, G., Griffiths, C., \& Krueger, J. (2015). Early intervention in psoriasis and immune-mediated inflammatory diseases: a hypothesis paper. J Dermatolog Treat, 103-12.

Gisondi, P., Conti, A., \& Galdo, G. (2013). Ustekinumab does not increase body mass index in patients with chronic plaque psoriasis: a prospective cohort study. Br J Dermatol, 1124-7.

Gisondi, P., Lora, V., \& Bonauguri, C. (2013). Serum chemerin is increased in patients with chronic plaque psoriasis and normalizes following treatment with infliximab. Br J Dermatol, 749-55.

Gonzalez-Cantero, A., Gonzalez-Cantero, J., \& Sanchez-Moya, A. (2019). Subclinical atherosclerosis 
in psoriasis. Usefulness of femoral artery ultrasound for the diagnosis, and analysis of its relationship with insulin resistance. PLOS ONE.

Hanauer, S., Sandborn, W., \& Rutgeerts, P. (2006). Human antitumor necrosis factor monoclonal antibody (adalimumab) in Crohn's disease: the CLASSIC-I trial. Gastroenterology , 323-33.

Hjuler, K., Gormsen, L., \& Vendelbo, M. (2017). Increased global arte-rial and subcutaneous adipose tissue inflammation in patients with moderate-to-severe psoriasis. Br J Dermatol, 732-40.

Joshi, A., Lerman, J., \& Aberra, T. (2016). GlycA is a novel biomarker of inflammation and subclinical cardiovascular disease in psoriasis. Circ Res, 1242-53.

Kim, B., Lee, W., \& Pak, K. (2019). Ustekinumab treatment is associated with decreased systemic and vascular inflammation in patients with moderate-to-severe psoriasis: Feasibility study using (18) F-fluorodeoxyglucose PET/CT. J Am Acad Dermatol , 1322.

Kolbinger, F., Loesche, C., \& Valentin, M. (2017). b-Defensin 2 is a responsive biomarker of IL-17Adriven skin pathology in patients with psoriasis. J Allergy Clin Immunol, 923-32.

Lerman, J., \& Joshi, A. (2017). Coronary plaque charac-terization in psoriasis reveals high-risk features that improve after treatment in a prospective observational study. Circulation, 263-76.

Low, A., Symmons, D., \& Lunt, M. (2017). Relationship between expo-sure to tumour necrosis factor inhibitor therapy and incidence and severity of myocardial infarction in patients with rheumatoid arthritis. Ann Rheum Dis, 654-60.

Lowes, M., Suarez-Farinas, M., \& Krueger, J. (2014). Immunology of psoriasis. Annu Rev Immunol, 22755.
Mehta, N., Shin, D., \& Joshi, A. (2018). Effect of 2 psoriasis treatments on vascular inflammation and novel inflammatory cardiovascular biomarkers: a randomized placebo-controlled trial. Circ Cardiovasc Imaging.

Nestle, F., Kaplan, D., \& Barker, J. (2009). Psoriasis. N Engl J Med, 361(1), 496-509.

Paller, A., Singh, R., \& Cloutier, M. (2018). Prevalence of psoriasis in chil-dren and adolescents in the United States: a claims-based analysis. J Drugs Dermatol , 187-94.

Reich, K. (2012). The concept of psoriasis as a systemic inflammation: implications for disease management. J Eur Acad Dermatol Venereol, 3-11.

Savage, L., Goodfield, M., Horton, \& L. (2018). Regression of peripheral subclinical enthesopathy in therapy-naive patients treated with ustekinumab for moderate-to-severe chronic plaque psoriasis. Arthritis Rheumatol, 626-31.

Shrivastava, A., Singh, H., Raizada, A., \& Singh, S. (2015). C-reative protein, inflammation and coronary heart disease. Egypt Heart J, 89-97.

Swindell, W., Johnston, A., \& Xing, X. (2013). Modulation of epidermal transcription circuits in psoriasis: new links between inflammation and hyperproliferation. PLOS ONE .

Takeshita, J., Grewal, S., \& Langan, S. (2017). Psoriasis and comorbid dis-eases: epidemiology. J Am Acad Dermatol, 377-90.

Wu, J., Guerin, A., \& Sundaram, M. (2017). Cardiovascular event risk assessment in psoriasis patients treated with tumor necrosis factor-alpha inhibitors versus methotrexate. J Am Acad Dermatol, 81-90.

\section{CITAR ESTE ARTICULO:}

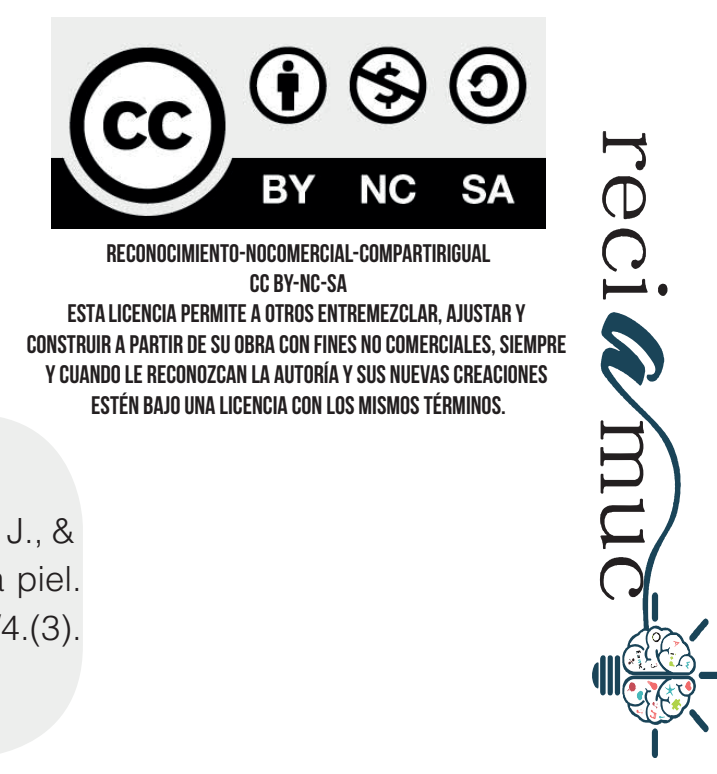

Carranza Delgado, K., Montenegro Villavicencio, A., Navarrete Moncayo, J., \& Sarmiento Hidalgo, B. (2020). La psoriasis como desorden común de la piel. Causas y tratamientos. RECIAMUC, 4(3), 38-49. doi:10.26820/reciamuc/4.(3). julio.2020.38-49 